\title{
Character Association and Path Coefficient Analysis for Grain Yield and Yield Components of Parents and Hybrids in Rice (Oryza sativa L.)
}

\author{
Ch. Ramesh*, Ch. Damodar Raju, Ch. Surender Raju and N. Ram Gopala Varma \\ Department of Genetics and Plant Breeding, Professor Jayashankar Telangana State \\ Agricultural University, College of Agriculture, Rajendranagar-500030 (TG), India
}

*Corresponding author

\section{A B S T R A C T}

\begin{tabular}{|l|}
\hline Ke y w o r d s \\
Rice (Oryza sativa \\
L.), Grain yield
\end{tabular}

\section{Introduction}

As rice is the staple food in most parts of India providing 43 per cent of calorie requirement for more than 70 per cent of Indian population and there is a need to increase production of rice and productivity of land under rice cultivation. India has the largest acreage under rice at $44 \mathrm{M}$. ha ( $22 \%$ of cropped area) with annual production of $106.65 \mathrm{MT}$ in the year 2013-14 as per Agricultural Statistics Division, Directorate of Economics and Statistics, Department of Agriculture and Cooperation. A thorough understanding of the interaction of characters among themselves had been of great use in plant breeding. The efficiency of selection for yield mainly depends on the direction and magnitude of association between yield and its component characters and also among themselves. Character association provides information on the nature and extent of association between pairs of metric traits and helps in selection for the improvement of the character.

Correlation gives only the relation between two variables whereas path coefficient analysis allows separation of the direct effect and their indirect effects through other attributes by partitioning the correlations (Wright, 1921). Path analysis is that, it permits the partitioning of the correlation coefficient into its components, one component being the path coefficient that measures the direct effect of a predictor variable upon its response variable; the second component being the 
indirect effect(s) of a predictor variable on the response variable through another predictor variable (Dewey and Lu, 1959). This study made an attempt to identify the genetic variability in the parents and the hybrids that are obtained by crossing them. Further an attempt was made to identify the major contributing characters for grain yield, their direct and indirect effects.

\section{Materials and Methods}

This study involves 35 genotypes which includes, 11 parents viz. RNR 21615, RNR 21604, RNR21304, RNR 21218, RNR 210301, RNR 21288, MTU 1010, RNR 15048, JMS 13A, IR 58025A and IR 68897A and 24 cross combinations was conducted at Rice Research Centre, Rajendranagar during Kharif-2015. The experiment for studying character association and their direct and indirect effects on grain yield was laid in Randomized Block Design (RBD) with three replications.

Thirty days old seedlings were transplanted in the main field and all the necessary package of practices were followed to raise a healthy crop. Observations were recorded on 16 characters viz., days to 50 per cent flowering, plant height, panicle length, panicle weight, number of productive tillers per plant, number of unproductive tillers per plant, flag leaf length, flag leaf width, spikelet fertility\%, number of filled grains per panicle, grain yield per plant, productivity/day, 1000 grain weight, grain length, grain breadth and grain lengthbreadth ratio and subjected to statistical analysis.

Statistical analysis and methodology used to conduct this study, for the above characters as per Singh and Chaudhary (1985) for correlation coefficient and Dewey and Lu (1959) for path analysis which were standard procedures used till today.

\section{Results and Discussion}

Phenotypic and genotypic correlations between yield and yield components viz., days to 50 per cent flowering, plant height, panicle length, panicle weight, number of productive tillers per plant, number of unproductive tillers per plant, flag leaf length, flag leaf width, spikelet fertility\%, number of filled grains per panicle, grain yield per plant, productivity/day, 1000 grain weight, grain length, grain breadth and grain length-breadth ratio were estimated. In general, genotypic correlations were found to be higher than phenotypic correlations, which indicate that though there is strong inherent association between characters studies, its expression is lessened due to influence of environment and considering the importance of phenotypic correlation it was discussed in the results which were presented in Table 1.

Days to 50 percent flowering recorded a significant positive phenotypic correlation with grain yield per plant, panicle weight, number of productive tillers per plant, flag leaf width, spikelet fertility $\%$ and number of filled grains per panicle. Bhadru et al., (2011), Patel et al., (2014) and Srijan et al., (2016) also reported significant positive phenotypic correlation of days to 50 per cent flowering with grain yield per plant. The plant height recorded a significant positive correlation with grain yield per plant, panicle length, panicle weight, number of productive tillers per plant, flag leaf width, number of filled grains per panicle and per day productivity and showed significant negative correlation with flag leaf length. The significant positive phenotypic correlation of plant height with grain yield per plant was also reported earlier by Bhadru et al., (2011), Eswara Reddy et al., (2013), Patel et al., (2014) and Srijan et al., (2016). Panicle length showed significant positive correlation with grain yield per plant, panicle weight, number of productive tillers / plant, flag leaf 
length, flag leaf width, spikelet fertility \%, number of filled grains / panicle, per day productivity, 1000 grain weight, grain length and grain breadth. Significant positive phenotypic correlation of panicle length with grain yield per plant was also reported by Bhadru et al., (2011) and Patel et al., (2014) and Srijan et al., (2016). Panicle weight recorded a significant positive correlation with grain yield per plant, number of productive tillers per plant, flag leaf width, spikelet fertility $\%$, number of filled grains per panicle, per day productivity and 1000 grain weight. Significant positive phenotypic correlation was reported by Bhadru et al., (2011) and Srijan et al., (2016). Number of productive tillers per plant recorded significant positive correlation with grain yield per plant, flag leaf width, spikelet fertility\%, number of filled grains per panicle, per day productivity and 1000 grain weight. In earlier positive significant correlation of number of productive tillers with number of filled grains per panicle was reported by Seyoum et al., (2012) and Patel et al., (2014). Sabesan et al., (2009), Patel et al., (2014) and Moosavi et al., (2015) also reported desirability of significant positive phenotypic correlation of number of productive tillers per plant, with grain yield per plant. Number of unproductive tillers per plant showed negative correlation with per day productivity and length / breadth ratio as reported by Srijan et al., (2016). Flag leaf length showed significant positive correlation with flag leaf width. Flag leaf width recorded significant positive correlation with grain yield per plant and per day productivity, spikelet fertility percentage and number of filled grains per panicle. While the significant negative correlation of Flag leaf width with spikelet fertility was reported by Srijan et al., (2016). Spikelet fertility \% recorded positive correlation with grain yield per plant, number of filled grains per panicle, per day productivity, 1000 grain weight, grain length and grain breadth. Negative correlation of spikelet fertility \% with grain yield was reported by Srijan et al., (2016).

Number of filled grains per panicle recorded significant positive correlation with grain yield per plant, per day productivity, grain length and grain breadth. Similar results were reported by Srijan et al., (2016). Per day productivity recorded positive correlation with grain yield per plant, 1000 grain weight and grain breadth. Bhadru et al., (2011) and Srijan et al., (2016) also reported significant positive phenotypic correlation of productivity per day with, grain yield per plant. 1000 grain weight showed positive correlation with grain yield plant, grain length and grain breadth as reported by Patel et al., (2014). Grain length showed positive correlation with grain breadth and grain length / breadth. Significant positive correlation of grain length with grain length/ breadth ratio was reported by Patel et al., (2014). Grain breadth showed significant positive correlation with grain yield per plant and grain length / breadth ratio, While negative correlation of grain breadth with grain length / breadth ratio was reported by Srijan et al., (2016) Grain length / breadth ratio showed significant positive correlation with number of unproductive tillers per plant, grain length and grain breadth where as positive significant positive correlation with grain length was reported by Srijan et al., (2016).

As simple correlation does not provide the true contribution of the characters towards the yield, these genotypic correlations were partitioned into direct and indirect effects through path coefficient analysis. It allows separating the direct effect and their indirect effects through other attributes by partitioning the correlations (Wright, 1921) for better interpretation of cause and effect relationship. The estimates of path coefficient analysis are provided for yield and yield component characters in Table 2. 


\section{Table.1}

\begin{tabular}{|c|c|c|c|c|c|c|c|c|c|c|c|c|c|c|c|c|c|}
\hline Trait & & dff & $\begin{array}{l}\text { PH } \\
\text { (Cm) }\end{array}$ & PL (Cm) & $\begin{array}{l}\text { P } \\
\text { WT(gm) }\end{array}$ & P TIL & $\begin{array}{l}\text { UP } \\
\text { TIL }\end{array}$ & FL (cm) & FW(cm) & S F $(\%)$ & FG/P & $\begin{array}{l}\text { PDP } \\
(\mathrm{gm} / \mathrm{D})\end{array}$ & $\begin{array}{l}1000 \mathrm{GW} \\
(\mathrm{gm})\end{array}$ & GL(mm) & GB(mm) & L/B & SP Y(gm) \\
\hline \multirow[t]{2}{*}{ dff } & $\mathrm{P}$ & 1.0000 & 0.1861 & 0.1673 & $0.4398^{* *}$ & $0.196^{*}$ & 0.0498 & -0.0251 & $0.3941^{* *}$ & $0.3336^{* *}$ & $0.312^{* *}$ & 0.1256 & 0.1562 & -0.0377 & 0.0805 & -0.0809 & $0.2327^{*}$ \\
\hline & G & 1.0000 & $0.2197 *$ & $0.2420^{*}$ & $0.5011^{* *}$ & $0.2730 * *$ & 0.0852 & -0.0336 & $0.4772 * *$ & $0.4000^{* * *}$ & $0.3580 * *$ & 0.1788 & $0.1819^{*}$ & -0.0588 & 0.0937 & -0.1100 & $0.2662 * *$ \\
\hline \multirow[t]{2}{*}{ PH (Cm) } & $\mathrm{P}$ & & 1.0000 & $0.3361 * *$ & $0.4480 * *$ & $0.1879 *$ & -0.0212 & $-0.2030 *$ & $0.2871 * *$ & 0.1836 & $0.5052 * *$ & $0.4089 * *$ & 0.1419 & 0.1103 & 0.1612 & -0.0256 & $0.4122 * *$ \\
\hline & G & & 1.0000 & $0.4645^{* *}$ & $0.4723 * *$ & $0.2027^{*}$ & 0.0051 & -0.2332 & $0.3313^{* *}$ & $0.1990^{*}$ & $0.5256^{* *}$ & $0.4283^{* *}$ & 0.1483 & 0.1111 & 0.1687 & -0.0294 & $0.4289 * *$ \\
\hline \multirow{2}{*}{ PL (Cm) } & $\mathrm{P}$ & & & 1.0000 & $0.3612 * *$ & $0.3781 * *$ & 0.1754 & $0.3129 * *$ & $0.2394 *$ & $0.3232 * *$ & $0.2590 * *$ & $0.3156^{* *}$ & $0.2520 * *$ & $0.2624 * *$ & $0.3149 * *$ & -0.0071 & $0.3280 * *$ \\
\hline & G & & & 1.0000 & $0.4154 * *$ & $0.4241 * *$ & 0.1825 & $0.4008 * *$ & $0.2637 * *$ & $0.3976^{* *}$ & $0.3306^{* *}$ & $0.3896^{* *}$ & $0.3085^{* *}$ & $0.3124 * *$ & $0.4001 * *$ & -0.0132 & $0.4011 * *$ \\
\hline \multirow[t]{2}{*}{ p wt } & $\mathrm{P}$ & & & & 1.0000 & $0.4130^{* *}$ & -0.1717 & 0.0751 & $0.2667 * *$ & $0.6011^{* *}$ & $0.7062 * *$ & $0.6107 * *$ & $0.3028 * *$ & 0.1422 & 0.1401 & 0.0261 & $0.6385^{* *}$ \\
\hline & $\mathrm{G}$ & & & & 1.0000 & $0.4607 * *$ & -0.2041 & 0.0828 & $0.2872^{* *}$ & $0.6128 * *$ & $0.7168 * *$ & $0.6217 * *$ & $0.3052 * *$ & 0.1442 & 0.1451 & 0.0301 & $0.6467 * *$ \\
\hline \multirow[t]{2}{*}{ P TIL } & $\mathrm{P}$ & & & & & 1.0000 & -0.0560 & 0.0091 & $0.3158 * *$ & $0.4844 *$ & $0.2391 *$ & 0.6520 ** & $0.2183 *$ & 0.0536 & 0.0517 & 0.0146 & $0.6695 * *$ \\
\hline & $\mathrm{G}$ & & & & & 1.0000 & -0.0642 & -0.0081 & $0.3814^{* *}$ & $0.5506^{* *}$ & $0.2792 * *$ & $0.7103^{* *}$ & $0.2357^{*}$ & 0.0530 & 0.0510 & 0.0143 & $0.7235^{* *} *$ \\
\hline \multirow[t]{2}{*}{ UP TIL } & $\mathrm{P}$ & & & & & & 1.0000 & 0.0681 & 0.0171 & 0.0664 & -0.1364 & 0.0831 & 0.1263 & -0.0835 & 0.1508 & $-0.1910 *$ & -0.0797 \\
\hline & G & & & & & & 1.0000 & 0.0867 & -0.0013 & 0.0902 & -0.1651 & -0.0969 & 0.1511 & -0.0809 & 0.1888 & -0.2091 & -0.0948 \\
\hline \multirow[t]{2}{*}{ FL $(\mathrm{cm})$} & $\mathrm{P}$ & & & & & & & 1.0000 & $0.2749 * *$ & 0.0388 & -0.0956 & -0.0270 & -0.1227 & 0.0176 & 0.0622 & -0.0303 & -0.0207 \\
\hline & $\mathrm{G}$ & & & & & & & 1.0000 & $0.3294 * *$ & 0.0373 & -0.1045 & -0.0262 & -0.1374 & 0.0196 & 0.0719 & -0.0340 & -0.0193 \\
\hline \multirow[t]{2}{*}{ FW(cm) } & $\mathrm{P}$ & & & & & & & & 1.0000 & $0.2649 * *$ & $0.3000 * *$ & $0.2833 * *$ & -0.0342 & -0.1347 & 0.0135 & -0.1316 & $0.3248 * *$ \\
\hline & $\mathrm{G}$ & & & & & & & & 1.0000 & $0.2864 * *$ & $0.3250 * *$ & 0.3056 & -0.0409 & -0.1522 & 0.0029 & -0.1423 & $0.3509 * *$ \\
\hline \multirow[t]{2}{*}{ S F $(\%)$} & $\mathrm{P}$ & & & & & & & & & 1.0000 & $0.5082 * *$ & $0.6280 * *$ & $0.3989 * *$ & $0.2502 * *$ & $0.3065 * *$ & 0.0078 & $0.6411 * *$ \\
\hline & G & & & & & & & & & 1.0000 & $0.5075^{* *}$ & $0.6364 * *$ & $0.4040 * *$ & $0.2545^{* *}$ & $0.3212 * *$ & 0.0120 & $0.6494 * *$ \\
\hline \multirow[t]{2}{*}{ FG/ $P$} & $\mathrm{P}$ & & & & & & & & & & 1.0000 & $0.6223 * *$ & 0.1099 & $0.2402 *$ & $0.2448 *$ & 0.0377 & $0.6378 * *$ \\
\hline & G & & & & & & & & & & 1.0000 & $0.6295^{* *}$ & 0.1103 & $0.2411^{*}$ & $0.2539 * *$ & 0.0387 & $0.6427 * *$ \\
\hline \multirow[t]{2}{*}{ PDP } & $\mathrm{P}$ & & & & & & & & & & & 1.0000 & $0.2209 *$ & 0.0850 & $0.3229 * *$ & -0.1723 & $0.9923 * *$ \\
\hline & G & & & & & & & & & & & 1.0000 & $0.2219^{*}$ & 0.0856 & $0.3343^{* *}$ & -0.1739 & $0.9954 * *$ \\
\hline \multirow[t]{2}{*}{$1000 \mathrm{GW}$} & $\mathrm{P}$ & & & & & & & & & & & & 1.0000 & $0.4238 * *$ & $0.4924 * *$ & -0.0139 & $0.2297 *$ \\
\hline & G & & & & & & & & & & & & 1.0000 & $0.4249 * *$ & $0.5059 * *$ & -0.0112 & $0.2298^{*}$ \\
\hline \multirow[t]{2}{*}{ GL(mm) } & $\mathrm{P}$ & & & & & & & & & & & & & 1.0000 & $0.2952 * *$ & $0.6570 * *$ & 0.0673 \\
\hline & G & & & & & & & & & & & & & 1.0000 & $0.2841 * *$ & $0.6749^{* *}$ & 0.0652 \\
\hline \multirow[t]{2}{*}{ GB(mm) } & $\mathrm{P}$ & & & & & & & & & & & & & & 1.0000 & $0.5186 * *$ & $0.3194 * *$ \\
\hline & G & & & & & & & & & & & & & & 1.0000 & -0.5135 & $0.3311^{* *}$ \\
\hline \multirow[t]{2}{*}{ L/B } & $\mathrm{P}$ & & & & & & & & & & & & & & & 1.0000 & -0.1847 \\
\hline & G & & & & & & & & & & & & & & & 1.0000 & -0.1888 \\
\hline
\end{tabular}

DFF - Days to 50\% flowering, PH-Panicle Height, PL-Panicle Length, PWT- Panicle Weight, PTIL- NO. of productive tillers, UPTIL-No. of Unproductive Tillers, FL-Flag Leaf length, FW-Flag Leaf width, SF\%- Spikelet Fertility \%, FG/P-No. of filled grains per panicle, PDP- per day productivity, 1000 GWT- 1000 grain weight, GL-Grain Length, GB-Grain breadth, L/B-Grain length-breadth ratio, SPY-single plant yield. Phenotypic residual effect-0.0149, Genotypic residual effect- 0.0515. P-Phenotypic level, G-Genotypic level, Bold Values-Direct effects, Normal Values-Indirect effects, ** Significance at $1 \%$, *Significance at $1 \%$. 
Table. 2

\begin{tabular}{|c|c|c|c|c|c|c|c|c|c|c|c|c|c|c|c|c|c|}
\hline Trait & & dff & $\begin{array}{l}\text { PH } \\
(\mathbf{C m})\end{array}$ & $\begin{array}{l}\text { PL } \\
(\mathbf{C m})\end{array}$ & $\begin{array}{l}\text { P } \\
\text { WT(gm) }\end{array}$ & P TIL & UP TIL & FL (cm) & $\mathbf{F W}(\mathrm{cm})$ & S F (\%) & FG/P & $\begin{array}{l}\text { PDP } \\
(\mathrm{gm} / \mathrm{D})\end{array}$ & $\begin{array}{l}1000 \text { GW } \\
(\text { gm) }\end{array}$ & GL(mm) & $\mathbf{G B}(\mathrm{mm})$ & L/B & SP Y (gm) \\
\hline \multirow[t]{2}{*}{ dff } & $\mathrm{P}$ & 0.1180 & -0.0011 & 0.0005 & -0.0121 & 0.0042 & -0.0004 & -0.0004 & -0.0027 & -0.0049 & 0.0075 & 0.1229 & 0.0023 & -0.0021 & -0.0057 & 0.0067 & $0.2327^{*}$ \\
\hline & G & 0.1020 & -0.0022 & 0.0013 & -0.0141 & 0.0001 & -0.0011 & -0.0005 & 0.0007 & -0.0070 & 0.0046 & 0.1786 & 0.0036 & 0.0020 & 0.0005 & -0.0022 & $0.2662 * *$ \\
\hline \multirow[t]{2}{*}{ PH (Cm) } & $\mathrm{P}$ & 0.0220 & -0.0057 & 0.0010 & -0.0123 & 0.0040 & 0.0002 & -0.0034 & -0.0020 & -0.0027 & 0.0121 & $0.4001 * *$ & 0.0021 & 0.0062 & -0.0115 & 0.0021 & $0.4122 * *$ \\
\hline & $\mathrm{G}$ & 0.0224 & -0.0102 & 0.0024 & -0.0133 & 0.0001 & -0.0001 & -0.0034 & 0.0005 & -0.0035 & 0.0068 & $0.4278 * *$ & 0.0030 & -0.0038 & 0.0009 & -0.0006 & $0.4289 * *$ \\
\hline \multirow[t]{2}{*}{$\overline{\text { PL (Cm) }}$} & $\mathrm{P}$ & 0.0197 & -0.0019 & 0.0029 & -0.0099 & 0.0080 & -0.0013 & 0.0052 & -0.0016 & -0.0047 & 0.0062 & $0.3088 * *$ & 0.0037 & 0.0148 & -0.0224 & 0.0006 & $0.3280 * *$ \\
\hline & $\mathrm{G}$ & 0.0247 & -0.0048 & 0.0053 & -0.0117 & 0.0001 & -0.0024 & 0.0058 & 0.0004 & -0.0070 & 0.0043 & $0.3891^{* *}$ & 0.0061 & -0.0107 & 0.0021 & -0.0003 & $0.4011 * *$ \\
\hline \multirow[t]{2}{*}{ PW (gm) } & $\mathrm{P}$ & 0.0519 & -0.0026 & 0.0010 & -0.0274 & 0.0088 & 0.0013 & 0.0012 & -0.0018 & -0.0088 & 0.0169 & $0.5976^{* *}$ & 0.0045 & 0.0080 & -0.0100 & -0.0022 & $0.6385^{* *}$ \\
\hline & G & 0.0511 & -0.0048 & 0.0022 & -0.0282 & 0.0001 & 0.0027 & 0.0012 & 0.0004 & -0.0108 & 0.0092 & $0.6210 * *$ & 0.0061 & -0.0049 & 0.0008 & 0.0006 & $0.6467 * *$ \\
\hline \multirow[t]{2}{*}{ P TIL } & $\mathrm{P}$ & 0.0231 & -0.0011 & 0.0011 & -0.0113 & 0.0212 & 0.0004 & 0.0002 & -0.0022 & -0.0071 & 0.0057 & $0.6381^{* *}$ & 0.0032 & 0.0030 & -0.0037 & -0.0012 & $0.6695^{* *}$ \\
\hline & G & 0.0279 & -0.0021 & 0.0022 & -0.0130 & 0.0003 & 0.0009 & -0.0001 & 0.0006 & -0.0097 & 0.0036 & $0.7095 * *$ & 0.0047 & -0.0018 & 0.0003 & 0.0003 & $0.7235^{* *}$ \\
\hline \multirow[t]{2}{*}{ UP TIL } & $\mathrm{P}$ & 0.0059 & 0.0001 & 0.0005 & 0.0047 & -0.0012 & -0.0073 & 0.0011 & -0.0001 & -0.0010 & -0.0033 & -0.0814 & 0.0019 & -0.0047 & -0.0107 & 0.0158 & -0.0797 \\
\hline & G & 0.0087 & -0.0001 & 0.0010 & 0.0058 & 0.0000 & -0.0134 & 0.0013 & 0.0000 & -0.0016 & -0.0021 & -0.0968 & 0.0030 & 0.0028 & 0.0010 & -0.0042 & -0.0948 \\
\hline \multirow[t]{2}{*}{ FL (cm) } & $\mathrm{P}$ & -0.0030 & 0.0012 & 0.0009 & -0.0021 & 0.0002 & -0.0005 & 0.0165 & -0.0019 & -0.0006 & -0.0023 & -0.0264 & -0.0018 & 0.0010 & -0.0044 & 0.0025 & -0.0207 \\
\hline & G & -0.0034 & 0.0024 & 0.0021 & -0.0023 & 0.0000 & -0.0012 & 0.0145 & 0.0005 & -0.0007 & -0.0013 & -0.0261 & -0.0027 & -0.0007 & 0.0004 & -0.0007 & -0.0193 \\
\hline \multirow[t]{2}{*}{ FW(cm) } & $\mathrm{P}$ & 0.0465 & -0.0016 & 0.0007 & -0.0073 & 0.0067 & -0.0001 & 0.0045 & -0.0069 & -0.0039 & 0.0072 & $0.2772 *$ & -0.0005 & -0.0076 & -0.0010 & 0.0109 & $0.3248 * *$ \\
\hline & G & 0.0487 & -0.0034 & 0.0014 & -0.0081 & 0.0001 & 0.0000 & 0.0048 & 0.0015 & -0.0050 & 0.0042 & $0.3052 * *$ & -0.0008 & 0.0052 & 0.0000 & -0.0029 & $0.3509 * *$ \\
\hline \multirow[t]{2}{*}{ S F $(\%)$} & $\mathrm{P}$ & 0.0394 & -0.0011 & 0.0009 & -0.0165 & 0.0103 & -0.0005 & 0.0006 & -0.0018 & -0.0146 & 0.0122 & $0.6146^{* *}$ & 0.0059 & 0.0141 & -0.0218 & -0.0006 & $0.6411 * *$ \\
\hline & G & 0.0408 & -0.0020 & 0.0021 & -0.0173 & 0.0002 & -0.0012 & 0.0005 & 0.0004 & -0.0176 & 0.0065 & $0.6357^{* *} *$ & 0.0080 & -0.0087 & 0.0017 & 0.0002 & $0.6494 * *$ \\
\hline \multirow[t]{2}{*}{ FG/ P } & $\mathrm{P}$ & 0.0368 & -0.0029 & 0.0007 & -0.0194 & 0.0051 & 0.0010 & -0.0016 & -0.0021 & -0.0074 & 0.0240 & $0.6089 * *$ & 0.0016 & 0.0135 & -0.0174 & -0.0031 & $0.6378 * *$ \\
\hline & G & 0.0365 & -0.0054 & 0.0017 & -0.0202 & 0.0001 & 0.0022 & -0.0015 & 0.0005 & -0.0089 & 0.0129 & $0.6287 * *$ & 0.0022 & -0.0082 & 0.0013 & 0.0008 & $0.6427 * *$ \\
\hline \multirow[t]{2}{*}{ PDP } & $\mathrm{P}$ & 0.0148 & -0.0023 & 0.0009 & -0.0168 & 0.0138 & 0.0006 & -0.0004 & -0.0019 & -0.0092 & 0.0149 & $0.9785^{* *}$ & 0.0033 & 0.0048 & -0.0230 & 0.0142 & $0.9923 * *$ \\
\hline & $\mathrm{G}$ & 0.0182 & -0.0044 & 0.0021 & -0.0175 & 0.0002 & 0.0013 & -0.0004 & 0.0005 & -0.0112 & 0.0081 & $0.9988^{* *}$ & 0.0044 & -0.0029 & 0.0017 & -0.0035 & $0.9954 * *$ \\
\hline \multirow[t]{2}{*}{ PW (gm) } & $\mathrm{P}$ & 0.0184 & -0.0008 & 0.0007 & -0.0083 & 0.0046 & -0.0009 & -0.0020 & 0.0002 & -0.0058 & 0.0026 & $0.2162^{*}$ & 0.0148 & 0.0239 & -0.0351 & 0.0011 & $0.2297 *$ \\
\hline & G & 0.0186 & -0.0015 & 0.0016 & -0.0086 & 0.0001 & -0.0020 & -0.0020 & -0.0001 & -0.0071 & 0.0014 & $0.2217^{*}$ & 0.0199 & -0.0145 & 0.0026 & -0.0002 & $0.2298^{*}$ \\
\hline \multirow[t]{2}{*}{ GL(mm) } & $\mathrm{P}$ & -0.0045 & -0.0006 & 0.0008 & -0.0039 & 0.0011 & 0.0006 & 0.0003 & 0.0009 & -0.0036 & 0.0058 & 0.0832 & 0.0063 & 0.0563 & -0.0210 & -0.0542 & 0.0673 \\
\hline & G & -0.0060 & -0.0011 & 0.0016 & -0.0041 & 0.0000 & 0.0011 & 0.0003 & -0.0002 & -0.0045 & 0.0031 & 0.0855 & 0.0085 & -0.0341 & 0.0015 & 0.0137 & 0.0652 \\
\hline \multirow[t]{2}{*}{ GB(mm) } & $\mathrm{P}$ & 0.0095 & -0.0009 & 0.0009 & -0.0038 & 0.0011 & -0.0011 & 0.0010 & -0.0001 & -0.0045 & 0.0059 & $0.3159 * *$ & 0.0073 & 0.0166 & -0.0712 & 0.0428 & $0.3194 * *$ \\
\hline & $\mathrm{G}$ & 0.0096 & -0.0017 & 0.0021 & -0.0041 & 0.0000 & -0.0025 & 0.0010 & 0.0000 & -0.0056 & 0.0033 & $0.3339 * *$ & 0.0101 & -0.0097 & 0.0052 & -0.0104 & $0.3311 * *$ \\
\hline \multirow[t]{2}{*}{ L/B } & $\mathrm{P}$ & -0.0095 & 0.0001 & 0.0000 & -0.0007 & 0.0003 & 0.0014 & -0.0005 & 0.0009 & -0.0001 & 0.0009 & -0.1686 & -0.0002 & 0.0370 & 0.0369 & -0.0826 & -0.1847 \\
\hline & G & -0.0112 & 0.0003 & -0.0001 & -0.0008 & 0.0000 & 0.0028 & -0.0005 & -0.0002 & -0.0002 & 0.0005 & -0.1737 & -0.0002 & -0.0230 & -0.0027 & 0.0203 & -0.1888 \\
\hline
\end{tabular}

DFF - Days to 50\% flowering, PH-Panicle Height, PL-Panicle Length, PWT- Panicle Weight, PTIL- NO. of productive tillers, UPTIL-No. of Unproductive Tillers, FL-Flag Leaf length, FW-Flag Leaf width, SF\%- Spikelet Fertility \%, FG/P-No. of filled grains per panicle, PDP- per day productivity, 1000 GWT- 1000 grain weight, GL-Grain Length, GB-Grain breadth, L/B-Grain length-breadth ratio, SPY-single plant yield. Phenotypic residual effect-0.0149, Genotypic residual effect- 0.0515. P-Phenotypic level, G-Genotypic level, Bold Values-Direct effects, Normal Values-Indirect effects, ** Significance at $1 \%$, *Significance at $1 \%$. 
Among all the characters productivity per day was the major contributor for grain yield followed by, number of productive tillers per plant, spikelet fertility $\%$, number of filled grains per panicle, days to $50 \%$ flowering and grain breadth. These characters showed positive direct effect for grain yield per plant. On the other hand grain length / breadth ratio, number of unproductive tillers per plant and flag leaf length. These characters showed negative direct effect for grain yield per plant. Days to $50 \%$ flowering had positive indirect effect with grain yield through panicle length, number of productive tillers per plant, number of filled grains per panicle, per day productivity, 1000 grain weight, grain length / breadth ratio. Plant height showed positive indirect effect with grain yield through days to $50 \%$ flowering, panicle length, number of productive tillers per plant, number of unproductive tillers per plant, number of filled grains per panicle, per day productivity, 1000 grain weight, grain length, grain length / breadth ratio.

Panicle length had positive indirect effect with grain yield through, days to $50 \%$ flowering, number of productive tillers per plant, flag leaf length, number of filled grains per panicle, per day productivity, 1000 grain weight, grain length and grain length / breadth ratio. Panicle weight had positive indirect effect with grain yield through, days to $50 \%$ flowering, panicle length, number of productive tillers per plant, number of unproductive tillers per plant, flag leaf length, number of filled grains per panicle, per day productivity, 1000 grain weight and grain length. Number of productive tillers per plant had positive indirect effect with grain yield through, days to $50 \%$ flowering, panicle length, flag leaf length, number of filled grains per panicle, per day productivity, 1000 grain weight and grain length. Flag leaf width had positive indirect effect with grain yield through, days to $50 \%$ flowering, panicle length, number of productive tillers per plant, flag leaf length, number of filled grains per panicle, per day productivity and grain length / breadth ratio. Spikelet fertility $\%$ had positive indirect effect with grain yield through, days to $50 \%$ flowering, panicle length, number of productive tillers per plant, flag leaf length, falg leaf width, number of filled grains per panicle, per day productivity, 1000 grain weight and grain length. Number of filled grains per panicle had indirect effect with grain yield through, days to $50 \%$ flowering, panicle length, number of productive tillers per plant, number of unproductive tillers per plant, per day productivity, 1000 grain weight and grain length. Per day productivity had positive indirect effect with grain yield through, days to $50 \%$ flowering, panicle length, number of productive tillers per plant, number of unproductive tillers per plant, panicle length, number of filled grains per panicle, 1000 grain weight, grain length and grain length / breadth ratio. 1000 grain weight had positive indirect effect with grain yield per plant through, days to $50 \%$ flowering, panicle length, number of productive tillers per plant, flag leaf width, number of filled grains per panicle, per day productivity, grain length and grain length / breadth ratio.

Grain length had positive indirect effect with grain yield per plant through, panicle length, number of productive tillers per plant, number of unproductive tillers per plant, flag leaf length, flag leaf width, number of filled grains per panicle, per day productivity, 1000 grain weight. Grain breadth had positive indirect effect with grain yield through, days to $50 \%$ flowering, panicle length, number of productive tillers per plant, flag leaf length, number of filled grains per panicle, per day productivity,1000 grain weight, grain length and grain length / breadth ratio. Grain length / breadth ratio had positive indirect with grin yield through, plant height, number of 
productive tillers per plant, number of unproductive tillers per plant, number of filled grains per panicle, grain length and grain breadth.

Bhadru et al., (2011) reported the positive direct effects of productivity/ day, days to 50 per cent flowering, grain length, number of productive tillers per plant, panicle weight, number of filled grains per panicle, 1000 grain weight, flag leaf length, number of unproductive tillers per plant, plant height, panicle length, grain breadth and grain lengthbreadth ratio and negative direct effects of flag leaf width and spikelet fertility percent, on grain yield per plant. Srijan et al., (2016) reported negative indirect effect of number of unproductive tillers per plant through the number of productive tillers per plant, spikelet fertility $\%$, per day productivity and grain breadth.

Number of unproductive tillers per plant had negative indirect effect with grain yield through number of productive tillers per plant, flag leaf width, spikelet fertility $\%$, number of filled grains per panicle, per day productivity, grain length and grain breadth. Flag leaf length had negative indirect effect with grain yield through days to $50 \%$ flowering, panicle weight, number of unproductive tillers per plant, flag leaf width, spikelet fertility \%, number of filled grains per panicle, per day productivity, 1000 grain weight and grain breadth.

The study of phenotypic correlation studies showed that selection of plants with greater panicle length, more panicle weight, more number of filled grains per panicle, more number of productive tillers per plant, high productivity per day, 1000 grain weight, grain breadth, more plant height and more days to 50 per cent flowering would result in improvement of yield. Path analysis revealed that number of productivity/ day, spikelet fertility\%, number of filled grains per panicle, number of productive tillers per plant, panicle weight, plant height, 1000 grain weight, flag leaf width are the most important characters which could be used as selection criteria for effective improvement of grain yield.

Therefore, it is suggested that preference should be given to these characters in the selection programmes, to isolate superior lines with genetic potentiality for higher yield in rice genotypes.

\section{References}

Bhadru, D., Lokanadha Reddy, D and Ramesha, M.S. (2011). Correlation and path coefficient analysis of yield and yield contributing traits in rice hybrids and their parental lines. Electronic Journal of Plant Breeding, 2(1): 112116.

Dewey, J.R and Lu, K.H. (1959). Correlation and path coefficient analysis of components of crested wheat grass seed production. Agronomy Journal, 51: 515518.

Ekka, R.E., Sarawgi, A.K and Kanwar, R.R. (2011). Correlation and path analysis in traditional rice accessions of Chhattisgarh. Journal of Rice Research, 4(1 \& 2): 1118 .

Eswara Reddy, G., Suresh B.G., Sravan, T and Ashok Reddy, P. (2013). Interrelationship and cause-effect analysis of rice genotypes in north east plain zone. The Bioscan, 8(4): 11411144.

Moosavi, M., Ranjbar, G., Zarrini, H.N and Gilani, A. (2015). Correlation between morphological and physiological traits and path analysis of grain yield in rice genotypes under Khuzestan conditions. Biological Forum, 7(1): 43-47.

Patel, J.R., Saiyad, M.R., Prajapati, K.N., Patel, R.A and Bhavani R.T. (2014). 
Genetic variability and character association studies in rainfed upland rice (Oryza sativa L.). Electronic Journal of Plant Breeding, 5(3): 531537. Sabesan, T., Suresh, R and Saravanan, K. (2009). Genetic variability and correlation for yield and grain quality characters of rice grown in coastal saline low land of Tamilnadu. Electronic Journal of Plant Breeding, 1: $56-59$.

Seyoum, M., Alamerew, S and Bantte, K. (2012). Genetic variability, heritability, correlation coefficient and path analysis for yield and yield related traits in upland rice (Oryza sativa L.). Journal of Plant Sciences, 1-10.

Singh, R.K. and Chaudhary, B.D. (1985). Biometrical methods in quantitative genetic analysis. Kalyani Publishers, New Delhi, Ludhiana, India: 205-215. Wright, S. (1921). Correlation and causation. Journal of Agricultural Research, 20: 57-585.

Srijan. A., Sudheer Kumar, S., Damodar Raju, Ch. and Jagadeeshwar, R. Character association and path coefficient analysis for grain yield of parents and hybrids in rice (Oryza sativa L.). Journal of Applied and Natural Science 8 (1): 167 -172 (2016).

\section{How to cite this article:}

Ramesh, Ch., Ch. Damodar Raju, Ch. Surender Raju and Ram Gopala Varma, N. 2018. Character Association and Path Coefficient Analysis for Grain Yield and Yield Components of Parents and Hybrids in Rice (Oryza sativa L.). Int.J.Curr.Microbiol.App.Sci. 7(04): 2692-2699. doi: https://doi.org/10.20546/ijcmas.2018.704.307 